This is an Accepted Manuscript of

M. Fantuzzi, D. Masotti and A. Costanzo, "A Novel Integrated UWB-UHF One-Port Antenna for Localization and Energy Harvesting"

in:

IEEE Transactions on Antennas and Propagation, vol. 63, no. 9, pp. 3839-3848, Sept. 2015

The final published version is available online at:

https://doi.org/10.1109/TAP.2015.2452969

Rights / License:

The terms and conditions for the reuse of this version of the manuscript are specified in the publishing policy. For all terms of use and more information see the publisher's website.

This item was downloaded from IRIS Università di Bologna (https://cris.unibo.it/)

When citing, please refer to the published version. 


\title{
A novel integrated UWB-UHF one-port antenna for localization and energy harvesting
}

\author{
Marco Fantuzzi, Diego Masotti, Member, IEEE, and Alessandra Costanzo, Senior Member, IEEE
}

\begin{abstract}
In this work a novel compact, single-port antenna combining both UWB and UHF bands is presented. This solution is proposed for next generation passive RFID tags, performing communication and localization through UWB technology and efficient energy harvesting in the UHF band, but it is also designed in order to keep compatibility with previous tags generations. The UWB communication is deployed by means of an Archimedean spiral topology, while energy harvesting at UHF is obtained by suitably extending the spiral outer arms, thus realizing a meandered dipole, without affecting the UWB behavior. The single-port architecture provides an overall size reduction, while preserving the radiation performance in both the operating bands. This solution allows a direct connection to future integrated UWB-UHF chips, to standard RFID chips, or to a diplexer for suitably combining the UWB and the UHF functionalities. A prototype of this new antenna, realized on a standard FR-4 substrate, is first presented to validate the proposed novel design. As a second step, the same architecture is scaled on a paper substrate in view of its fully eco-compatible realization to be exploited by future pervasive RFID applications. The compact design of the antenna feeding network in discrete technology is also presented.
\end{abstract}

Index Terms-Ultra wideband antennas, UHF antennas, rectennas, radiofrequency energy harvesting, radiofrequency identification.

\section{INTRODUCTION}

$\mathrm{T}$ he fast growing evolution of our society, together with the development of information technologies, are inexorably leading us towards the introduction of systems more and more distributed in the environment. Terms like Internet of Things, Ubiquitous Electronics and Autonomous Logistics are therefore increasing their popularity.

The impact of systems organized as networked low-cost nodes (tags) that are distributed in the space, identifiable and localizable can be potentially enormous in a high number of

Manuscript received April 14, 2014. This work was partly funded by the Italian Ministry of the Instruction, University and Research (MIUR), within the framework of the PRIN-GRETA 2012 project.

M. Fantuzzi and D. Masotti are with DEI, University of Bologna, Bologna, Italy (corresponding author +39-051-2093792; fax: +39-051-2093053; e-mail: diego.masotti@unibo.it).

A. Costanzo is with DEI, University of Bologna, Cesena Campus, Italy (alessandra.costanzo@unibo.it).

(C) 2015 IEEE. Personal use of this material is permitted. Permission from IEEE must be obtained for all other uses, in any current or future media, including reprinting/republishing this material for advertising or promotional purposes, creating new collective works, for resale or redistribution to servers or lists, or reuse of any copyrighted component of this work in other works.

doi: 10.1109/TAP.2015.2452969 applications: e.g., logistics, security, energy and healthcare. In this context technologies such as radio-frequency identification (RFID) and real-time locating systems (RTLS) are of primary importance. However, the functionalities of identification and localization are typically offered by different wireless sub-systems. The possibility to combine them in a unique, highly efficient and possibly eco-friendly tag-device is therefore very attractive.

In the last years impulse-radio ultra-wideband (IR-UWB) technologies have demonstrated to be a promising solution to indoor localization problems, with sub-meter precision [1-4], thanks to their interference robustness characteristics. Thus a promising evolution of next-generation RFID is based on the exploitation of the UWB technology. The idea of backscattering UWB communication has been proposed for its use in low cost, semi-passive [1, 2, 5, 6], and passive tags [7]. Furthermore one of the advantages in adopting UWB communication is the ultra-low power consumption, which makes it possible to deploy battery-less RFID sensors by exploiting radiofrequency (RF) energy harvesting. For energy harvesting purposes UWB technology is not adoptable due to the extremely low spectral power density allowed. A possible solution, to keep the same radiating element for both communication and energy harvesting, could be the exploitation of the $2.45 \mathrm{GHz}$ band, which is close to the lower limit of the UWB band. However this would limit the maximum reachable link distances. This is clearly shown in Fig. 1 where the DC power delivered to the optimum load of two rectennas (rectifying antennas) operating at $900 \mathrm{MHz}$ and $2450 \mathrm{MHz}$, are plotted versus the free-space distance from the $\mathrm{RF}$ source. In both cases we consider an effective radiated power of $500 \mathrm{~mW}$, incident in the maximum link direction. Both the antennas are connected to a full-wave diode rectifier through a suitable matching network [8].

The great advantage in collecting RF energy from the ambient by exploiting the UHF band is clear: roughly one order of magnitude less in terms of rectified power is achieved at $2450 \mathrm{MHz}$. Thus, the best solution is to combine two radiating elements: one designed for energy harvesting purposes in the UHF band, the other one to sustain UWB communication. Moreover, this choice has the great advantage of guaranteeing full compatibility with previous RFID 
generations, as strongly recommended by market needs. This also allows the integration of UWB-RFID communication with ultra-low power sensors, autonomously sustained by the RF energy harvesting activity [8].

A possible realization on eco-friendly materials enables the

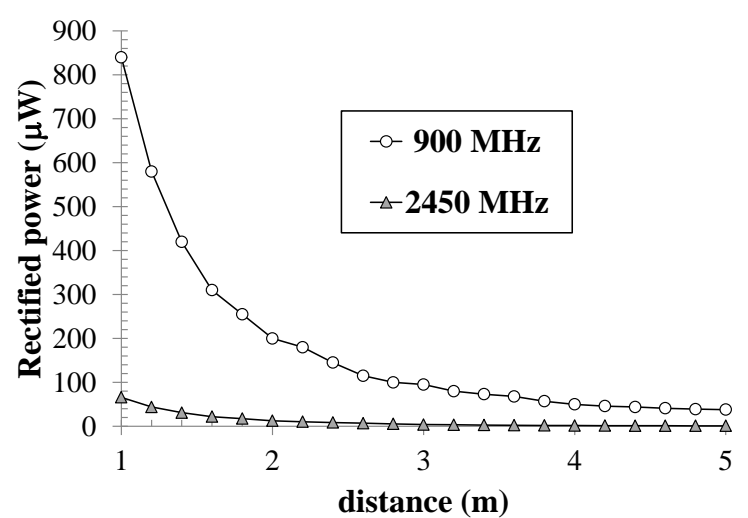

Fig. 1. Simulated DC converted output power for two rectennas, operating at 900 and $2450 \mathrm{MHz}$.

deployment of a distributed low-power intelligence (zero power smart tags) as envisaged by the "better society" chapter of Horizon $2020[9,10]$.

Hybrid UWB-UHF RFID systems have been already investigated [11] and several solutions of co-localization of UWB and UHF antennas are present in literature [12-14]. Most of them consist of smart placement of separate antennas on the same substrate, typically a UWB monopole/patch and a UHF dipole [12] or a $2.45 \mathrm{GHz}$ monopole [13], or a more compact slotted UHF dipole obtained from the ground plane of the UWB monopole [14]. However all these solutions offer two separate antennas architectures with two different ports, one for UWB (backscattered) communication, the other for UHF communication/harvesting.

With this paper we present the first single-port antenna solution for hybrid UWB-UHF systems operating in the European UHF-RFID and lower UWB bands: $868 \mathrm{MHz}$, and 3.1 to $4.8 \mathrm{GHz}$, respectively. In this way a superior size reduction with respect to previous solutions is achieved, maintaining a high quality of the radiation characteristics.

In order to validate design results, a first prototype of the proposed topology is realized on a standard FR-4 substrate. In view of the pervasive diffusion of "green" electronics, a second paper-based prototype is also realized and experimentally validated. A compact design of a three-port feeding network in microstrip technology is also provided to demonstrate the overall UWB-UHF system feasibility.

\section{HYBRID UWB-UHF ANTENNA DESIGN}

As in all wideband applications, an almost uniform antenna behavior in the whole frequency band, from the near-field (antenna impedance) and far-field points of view, is an important design requirement, though not always satisfied by the available solutions. Moreover, circular polarization is desirable in RFID applications to reduce tag installation
A large number of wideband antennas is available from literature. The simplest choice is to resort to step-wise [15] or tapered $[14,16,17]$ planar monopole topologies, with partial ground plane for image theorem application, or similarly inspired solutions, such as the Vivaldi antenna [17]. Many drawbacks pertain to these antennas: the dispersive behavior in terms of both far- and near-field, the linear polarization of the radiated field, and the unbalanced nature of the port (less suitable to chip connection). The antipodal Vivaldi antenna [18] solves the problem of the unbalanced port, only.

For the present design we exploit the self-complementary architecture [19] which theoretically provides an almost constant port impedance behavior in the whole band (ideal $\mathrm{Z} \approx$ $188.5 \Omega$, practical $\mathrm{Z} \approx 120 \Omega$, due to finite metallization thickness and feed line [19]) and identical radiation properties, since a fixed-shape "active zone" moves along the structure, by varying the operating frequency. Within this family of antennas, some topological choices permit to satisfy the circular polarization constraint, too: as the cases of standard Archimedean [19] and logarithmic spiral [17] topologies, both providing a balanced port, or a tapered version of the Archimedean spiral with unbalanced port [20].

The selected topology for the present design is the Archimedean spiral, providing the minimum size. This choice has been taken by comparison of electromagnetic (EM) simulations of an Archimedean and a logarithmic spiral antenna printed on a $1.5 \mathrm{~mm}$-thick FR-4 substrate $\left(\varepsilon_{\mathrm{r}}=4.3\right.$, $\tan (\delta)=0.025 @ 10 \mathrm{GHz})$, offering a similar behavior.

\section{A. Optimization and sizing of the Archimedean spiral antenna}

A first coverage of the UWB band, from 3.1 up to $4.8 \mathrm{GHz}$, is obtained by a $3.2 \mathrm{~cm}$-diameter spiral, with lines width (equal to lines gap for the auto-complementarity principle) of $1 \mathrm{~mm}$. The "active zone" of this antenna is the circular portion of the spiral having a circumference equal to the working wavelength $\lambda$ : in this section an in-phase condition for the currents flowing in the two stripes is reached, thus providing a behavior equivalent to a full-wavelength loop antenna [19].

It is well known that superior performance is achieved by thin-arms spiral antenna: the exploitation of vertical metallic strips (i.e. strips lain on the thin thickness side) allows to have much tighter turns: this way the number of strip turns can be considerably increased and thus a purer spiral behavior can be achieved [21]. A further goal, however, is to accomplish an additional UHF band coverage by means of a unique, compact, planar structure. As a first attempt in this direction, the above-described Archimedean spiral can be used by simply increasing its dimension; however this solution requires a spiral diameter of almost $13 \mathrm{~cm}$ to reach good radiation properties at the desired frequency, thus obtaining a total area incompatible with almost all RFID applications. This result clearly points out the necessity to investigate adhoc solutions in order to obtain the demanded goal within 
suitable overall dimensions.

Antenna miniaturization has always been of great interest in both commercial and military applications, therefore several techniques and guidelines have been proposed in literature $[22,23]$. A possible solution would be that of using high dielectric constants [24] or even magneto-dielectric [25] materials, but our final aim of realizing an eco-friendly device forces us to discard these possibilities. We thus resort to another miniaturization solution, the so-called "gap-loading" technique [26]: the inclusion of a capacitive load (a $1 \mathrm{~mm}$ circular stripe, placed $1 \mathrm{~mm}$-far from the spiral) increases the electrical length of the structure, thus reducing its overall dimension. However the almost $23 \%$ reduction factor reached is not yet enough for the desired purpose.

\section{B. Novel UWB and UHF antennas co-localization topology}

A new solution for co-localizing a UWB and a UHF antenna sharing the same substrate and the same port can be obtained by extending the spiral length to form a meandered dipole. In Fig. 2 the principle of the presented idea is shown; the reference system adopted in the whole paper is also shown in the same figure.

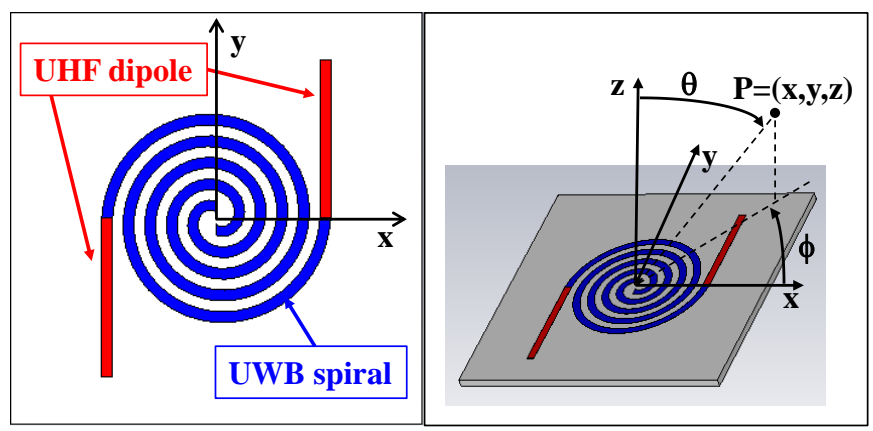

Fig. 2. New UWB-UHF antenna architecture.

The new topology justifies, from another point of view, the choice of the Archimedean spiral, instead of the logarithmic one: the fusion of the spiral with the dipole can be straightforwardly obtained without any tapering in-between the two structures. It is worth noting that all the spiral paths contribute to the dipole antenna, thus allowing an overall dipole length suitable for UHF band applications. It must be stressed that the UHF dipole still offers linear polarization, as standard straight dipoles: in fact, the far-field polarization is determined by the vertical arms departing from the spiral, while the rest of the branches mainly contribute as reactive loads. This is demonstrated by both EM-simulations and measurements in Section III. Linear polarization of the UHF antenna is required by the wireless powering mechanism foreseen in the National Project for which this antenna has been developed [10]: this is based on a set of intentional, linearly polarized UHF sources deployed in the ambient to provide the RF power on demand. Conversely, circular polarization for the UWB antenna is chosen for communication/ranging purposes, as usually required in most RFID applications.

In order to preserve the self-complementarity of the spiral

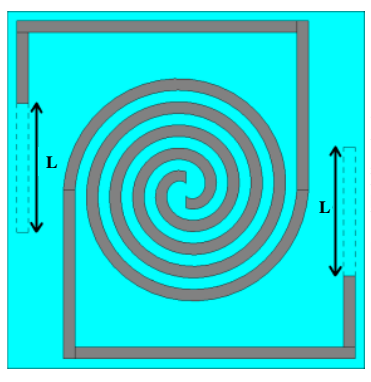

(a)

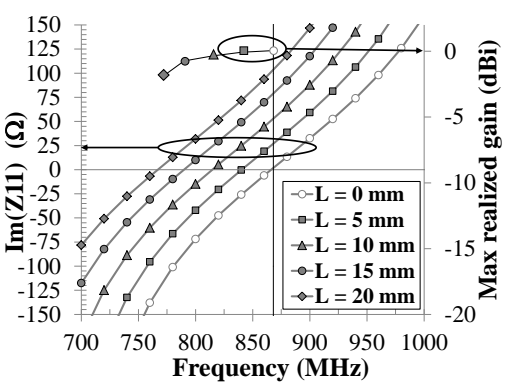

(b)
Fig. 3. Final topology of the proposed antenna (a), reactance and maximum realized gain as a function of the length of the dipole (b).

and to minimize lines coupling in the long path providing the dipole operation, wider lines with respect to previous experiments are chosen: lines and gaps width is set to $1.5 \mathrm{~mm}$.

This value has been chosen as a good compromise to guarantee the best spiral and dipole coexistence. Indeed this width leads to a total UHF dipole length of a 1.5- $\lambda$ UHF dipole while preserving a compact layout. Conversely, reduced width size better ensures the spiral currents in-phase condition [21] (with the number of turns increased accordingly, to preserve the outer circumference dimension). However this choice directly lengthens the UWB spiral arms and forces the use of a $2.5-\lambda$ UHF dipole, as the whole spiral path contribute to the dipole antenna. By EM simulation [27] we proved that this configuration worsens dipole performance, since most of its length comes from the spiral path. As a consequence, its polarization is turned from perfectly linear to elliptical. Moreover, longer and thinner paths involve higher losses, which would be a critical aspect in view of an ultra-low power, eco-compatible realization on paper substrate.

Finally, a standard $0.5-\lambda$ dipole cannot be adopted as well, since it would require an excessive reduction of the number of turns at the expense of the spiral performance, especially in the lower UWB frequency range.

The final topology of the antenna is reported in Fig. 3(a), where a picture of the prototype is shown. The folded dipole layout guarantees a resonant behavior at $868 \mathrm{MHz}$ (imaginary part of the dipole impedance equal to zero). However if the antenna is used with a standard UHF-RFID chip (for compatibility purposes with previous generations), an easy conjugate matching can be obtained by varying the final part of the dipole length (L) of Fig. 3(a). This is clearly shown in Fig. 3(b), where the imaginary part of the dipole input impedance is plotted versus frequency, with $\mathrm{L}$ as a parameter. It can be observed that a $20-\mathrm{mm}$ length variation provides a $100 \Omega$ reactance span. From Fig. 3(b) it is also clear that, for any selected length value, the reactance slope with respect to frequency does not vary significantly. This allows us to conclude that the operating RFID UHF bandwidth, shown later on in Fig. 10 (imaginary part $\approx 0$ ), can be guaranteed for many different dipole lengths. In Fig. 3(b) the corresponding realized gain, in resonant conditions, is also plotted: its almost flat behavior confirms that, by varying the branch $\mathrm{L}$ of the (C) 2015 IEEE. Personal use of this material is permitted. Permission from IEEE must be obtained for all other uses, in any current or future media, including reprinting/republishing this material for advertising or promotional purposes, creating new collective works, for resale or redistribution to servers or lists, or reuse of any copyrighted component of this work in other works. doi: 10.1109/TAP.2015.2452969 
total dipole length, the radiation properties in the UHF band are not significantly degraded. Thus these tuning properties can be exploited for compatibility with standard RFID chips and for the combined UWB-UHF applications addressed in

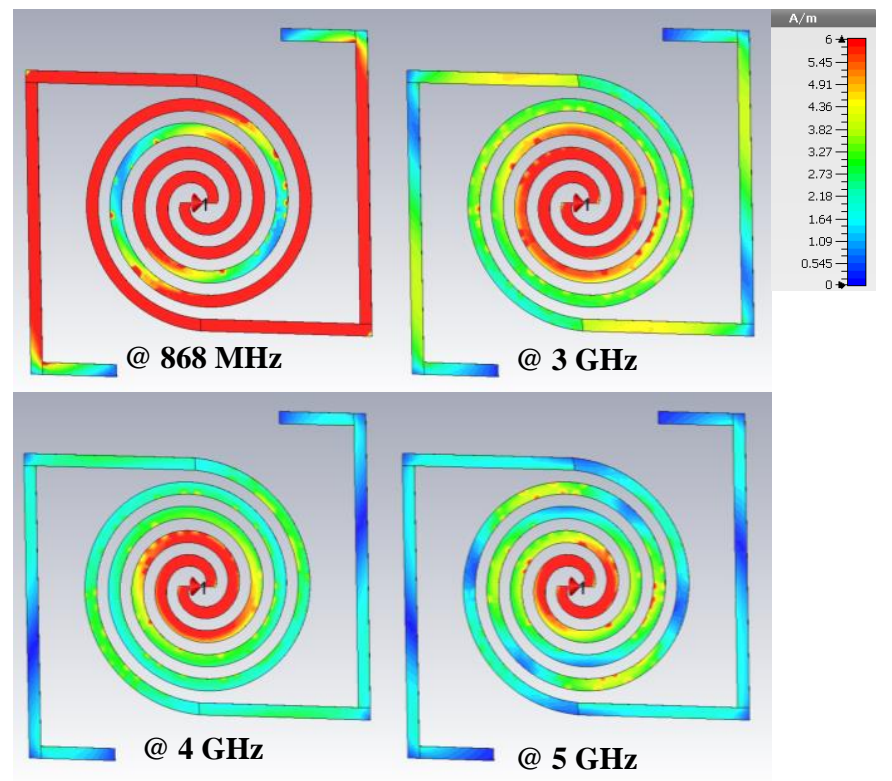

Fig. 4. Full-wave simulation results in terms of surface current magnitude in the UHF and UWB bands (higher values in red, lower in blue).

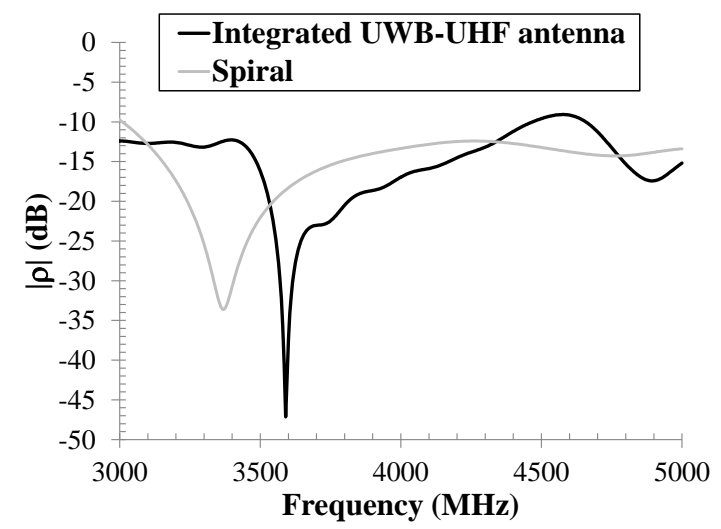

Fig. 5. Antenna performance in terms of reflection coefficient, without dipole (grey line) and with dipole (black line).

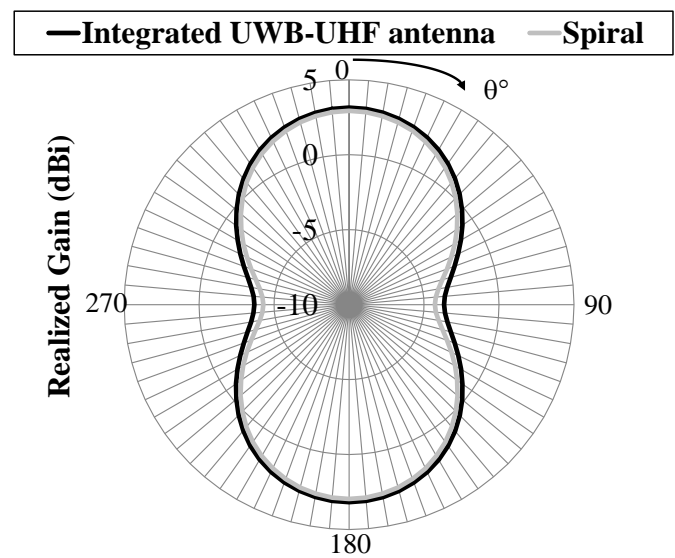

Fig. 6. Antenna performance in terms of realized gain in the yz-plane at the central UWB band frequency of $4 \mathrm{GHz}$, without dipole (grey line) and with dipole (black line).

reuse of any copyrighted component of this work in other works. doi: 10.1109/TAP.2015.2452969

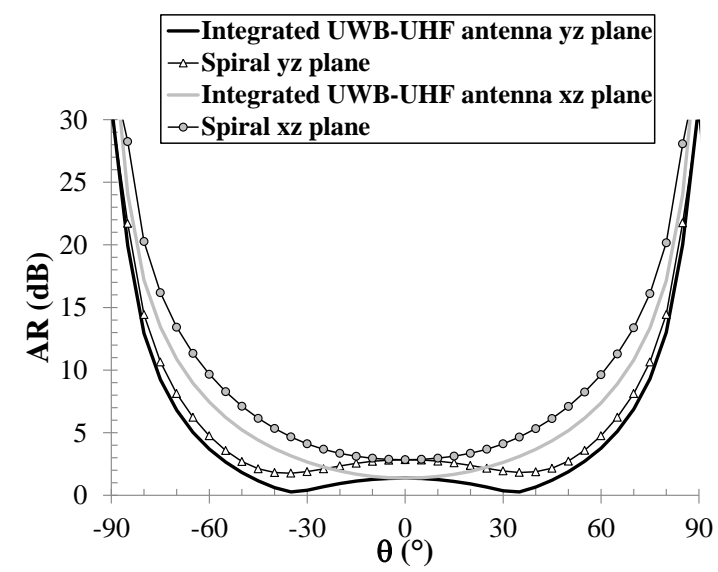

Fig. 7. Antenna simulated axial ratio at the central UWB band frequency of 4 $\mathrm{GHz}$, with and without dipole, in the $\mathrm{yz}-$ and $\mathrm{xz}$-plane.

this paper.

The first demonstration of the effectiveness of the presented idea is reported in Fig. 4, where the surface current magnitude, computed by full-wave simulation in correspondence of the operating frequencies is assessed. Besides the shift of the active zone within the UWB band, the 1.5- $\lambda$ behavior in the UHF band can be noticed, too: indeed two zero current values for each branch are evident.

A further confirmation of the optimal co-existence of the two antennas is then provided by the highly similar spiral performance with and without the dipole. In Fig. 5 the reflection coefficient (normalized to $120 \Omega$ ) is shown: a maximal value of $-10 \mathrm{~dB}$ is guaranteed for the two configurations in the frequency bands of interest. In Fig. 6 the plots of the radiation patterns in the yz-plane at the UWB center-frequency are compared, showing an excellent agreement. The same is true for the antenna circular polarization properties: in Fig. 7 the axial ratio (AR) in the xzand yz-plane of the UWB antennas are compared: a maximum difference of $1.5 \mathrm{~dB}$ is predicted in both planes. Similar results have been obtained all over the 3 to $5 \mathrm{GHz}$ band, thus ensuring the proper UWB communication in the presence of the UHF dipole.

Previous results also justify the insensitivity of the UWB antenna near- and far-field properties to dipole length variation, as verified by EM simulation.

\section{HYBRID UWB-UHF ANTENNA EXPERIMENTAL CHARACTERIZATION}

\section{A. FR-4 realization}

The new UWB-UHF integrated antenna has been first realized on a FR-4 substrate: the photo of the prototype with the associated dimensions is shown in Fig. 8(a).

As regards antenna port measurements we have to face the balanced nature of the antenna port. We resort to the measurement procedure proposed in [28], where a Y-shaped three-ports coaxial junction is used. After applying the cable de-embedding procedure we obtain the excellent 


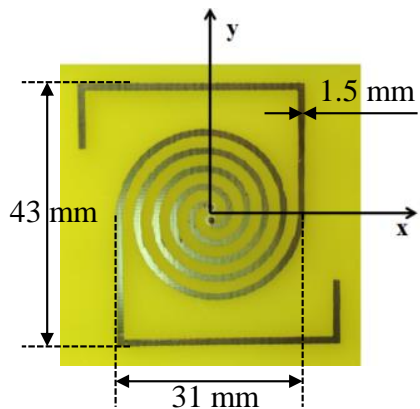

(a)

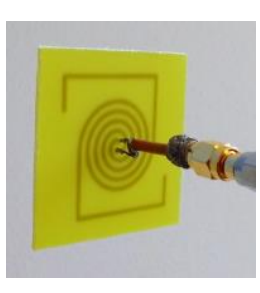

(b)
Fig. 8. Photo of the FR-4-based prototype: (a) front view; (b) perspective view with the coaxial cable used in radiation pattern measurements.

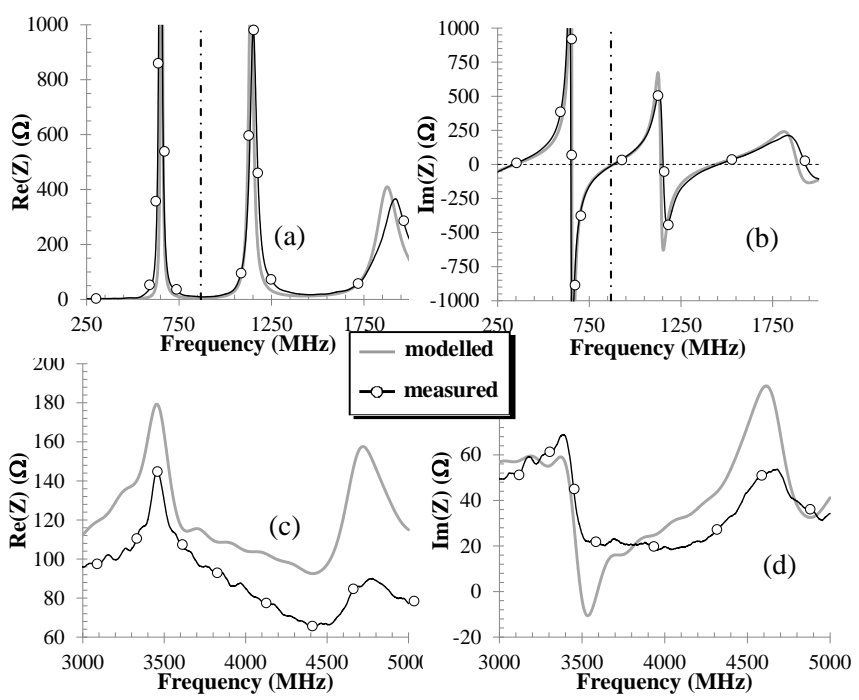

Fig. 9. Measured and modelled antenna input impedance: (a) real part, (b) imaginary part in the UHF band; (c) real part, (d) imaginary part in the UWB band.

correspondence between measured and modelled antenna impedance shown in Fig. 9, spanning both the UHF and the UWB bands. From inspection of the figure the exact resonance at $868 \mathrm{MHz}$, and the almost constant behavior in the UWB band can be easily evinced; the third zero crossing of the reactance in the lower band clearly confirms that the total length of the dipole provides a $1.5-\lambda$ behavior. It can be observed that in the UHF band the integrated antenna shows an impedance real part of about $12 \Omega$, which is acceptable for being matched to an RFID rectifier. This also confirms that a common half-wavelength topology is not adoptable due to its extremely low impedance values.

Very good agreement can also be observed by comparison of the modelled and measured reflection coefficients in both the UHF and UWB bands, by resorting to the proper normalization, as shown in Figs. 10 and 11, respectively. From the measurements of Fig. 10 the operating UHF bandwidth can be approximately derived as the frequency band where the magnitude of the reflection coefficient, normalized to the antenna impedance at resonance $(12 \Omega)$, is lower than $-10 \mathrm{~dB}$. A $35 \mathrm{MHz}$-band is achieved, thus providing compatibility with

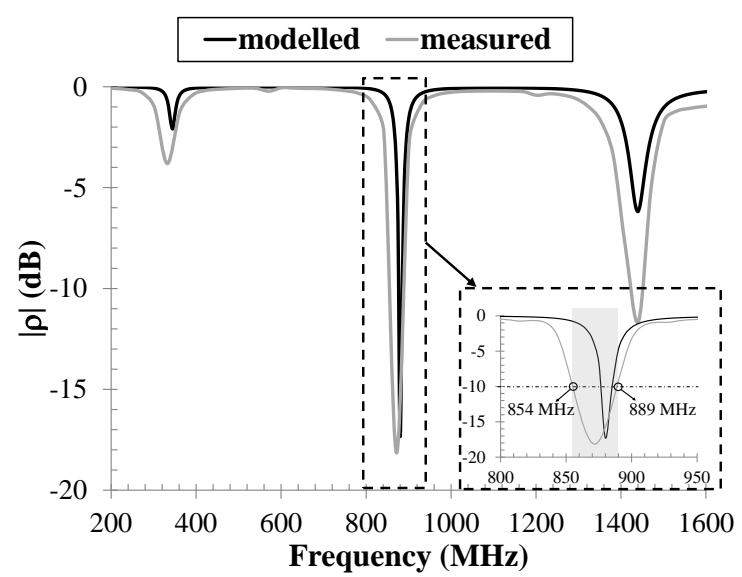

Fig. 10. Measured and modelled reflection coefficient in the UHF band (normalized to $12 \Omega$ ). $0.5-\lambda, 1.5-\lambda$ and $2.5-\lambda$ resonances can be clearly recognized.

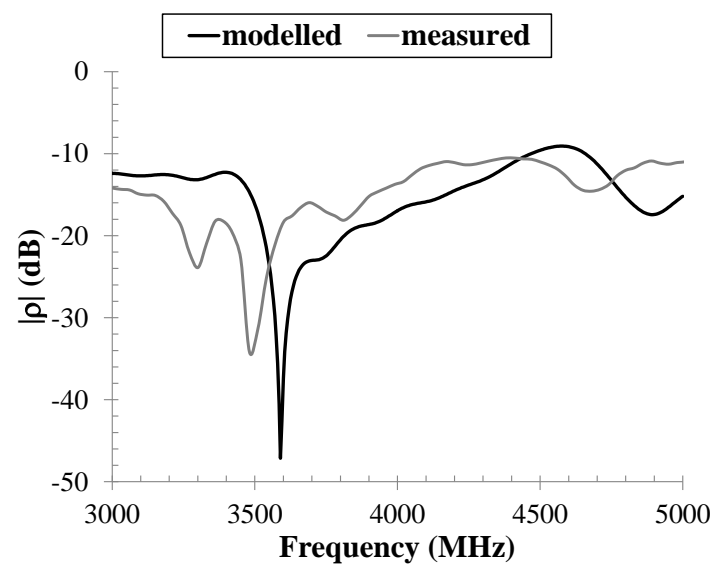

Fig. 11. Measured and modelled reflection coefficient in the UWB band (normalized to $120 \Omega$ ).

RFID chip practical band requirements. It is noteworthy that this is a fairly good bandwidth estimation, given that a rigorous one is not possible due to the nonlinear behavior of the antenna load (e.g. RFID chip) [29]. An alternative approximate estimation could be obtained by reducing the nonlinear chip input impedance to an equivalent RC circuit and considering the conjugate matching condition.

The highly dispersive behavior of the antenna impedance shown in Fig. 9 (especially by comparing the UHF and the UWB bands) must be accurately taken into account for the foreseen strategic tag realization. A UWB-UHF antenna feeding network can then be designed with the twofold goal of decoupling the UHF and UWB paths, and providing matching conditions, to a rectifier at $868 \mathrm{MHz}$ and to a backscattering modulator in the UWB band. An accurate description of its design in hybrid microstrip/lumped-element technology is given in Section IV.

Antenna far-field measurements are carried out in a real office scenario, and not in an anechoic chamber, in order to test the antenna performance in practical applications. Since we look for normalized behavior of the radiation properties, direct connection of the conductors of a 50- $\Omega$ coaxial cable 


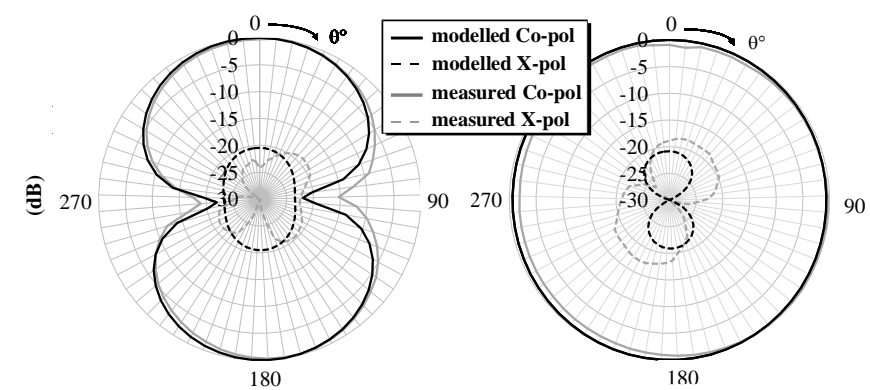

Fig. 12. Measured and modelled normalized field radiation patterns of the co- and cross-polarized component at $868 \mathrm{MHz}$, in the E-plane (yz) (a), and in the H-plane (xz) (b) of the dipole.

$[30,31]$ to the antenna differential port is adopted.

In Fig. 12 the predicted and measured radiation patterns of the co-polarized and cross-polarized components in the $\mathrm{E}$ and H-plane (yz- and xz-plane, respectively) of the UWB-UHF antenna at $868 \mathrm{MHz}$ are reported. As can be evinced, they prove to be almost identical to those obtained by a standard straight standalone dipole. The unique difference with respect to a straight dipole is the absence of secondary lobes, despite the geometrical 1.5- $\lambda$ length, since most of its total length is arranged in a spiral-like configuration. As regards the polarization of the dipole far-field, it results perfectly linearlypolarized in the y-direction (main axis of the dipole) for a wide elevation range. The light asymmetry of the radiation patterns of Fig. 12 is due to the coaxial cable termination
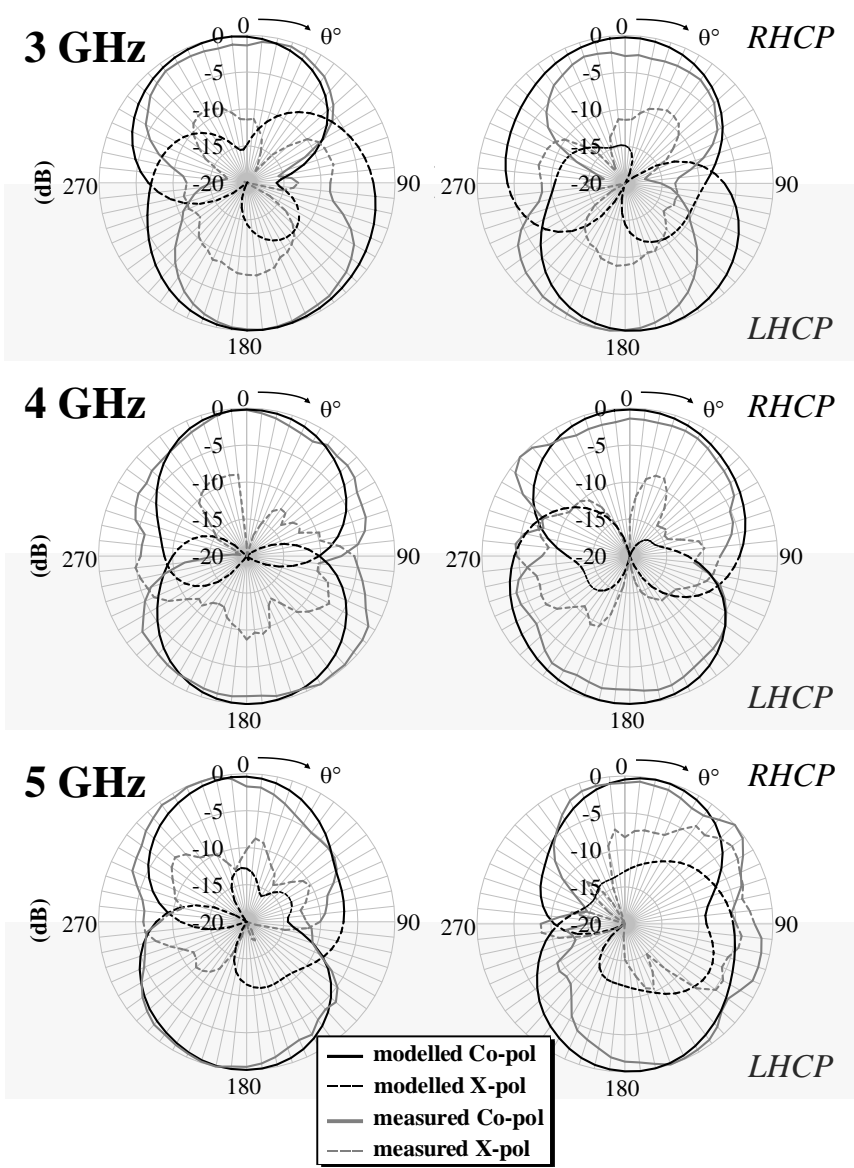

Fig. 13. Measured and modelled normalized field radiation patterns at 3, 4 and $5 \mathrm{GHz}$, in the yz-plane (left column), and in the xz-plane (right column). shown in Fig. 8(b), which is taken into account in the simulation, too. Similar conclusions can be drawn in the UWB band: Fig. 13 reports the simulated and measured radiation patterns at the UWB-band frequencies 3,4 and $5 \mathrm{GHz}$, for both the co- and the cross-polarized components. Due to the ungrounded antenna structure, in the $\mathrm{z}>0$ half-space the copolarized component is the right-handed circularly polarized (RHCP) one, while in the $\mathrm{z}<0$ half-space (light grey area in the figure) the co-polarized component is the left-handed circularly polarized (LHCP) one. The coaxial cable junction in the backside of the antenna is the main responsible of a not-

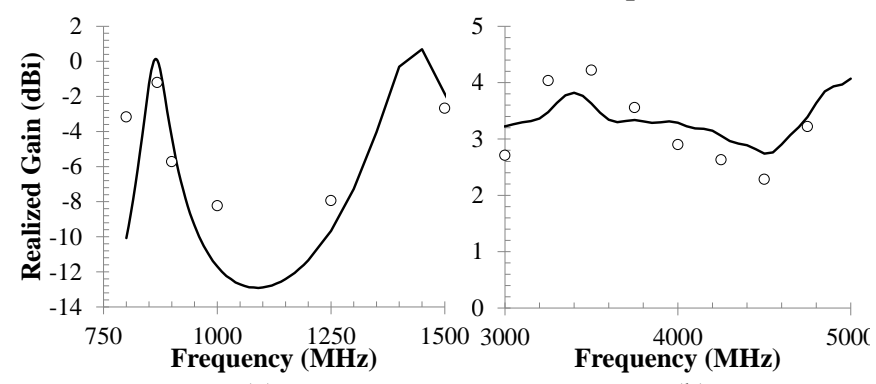

(a)

(b)

Fig. 14. Simulated (solid line) and measured (markers) realized gain of the FR4-prototype: (a) in the UHF band, (b) in the UWB band.

negligible cross-polarized component, as verified by simulation, too, and consequently of an AR worsening with respect to the isolated antenna results of Fig. 7.

The simulated realized gain in the maximal radiation direction of the antenna is plotted in Fig. 14, in both the operating frequency bands: an almost constant behavior is obtained in the UWB band, as expected; and a value of more than $0 \mathrm{dBi}$ is guaranteed in the UHF band. In the same figure the measured gain is superimposed, too: good agreement with modelled results is achieved.

These results were obtained using a $12 \Omega$ and a $120 \Omega$ termination, for the UHF and the UWB bands respectively.

\section{B. Paper-based realization}

In addition to the FR-4 solution, a second prototype of this innovative antenna has been realized on paper, taking into account the full compatibility with the environment. Paper is indeed an excellent candidate to reduce environmental impact of electronic circuits: it is widely available at a very low cost and most of all its highly biodegradability, with respects to

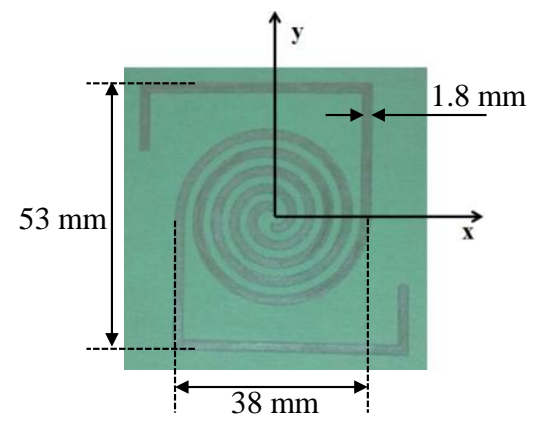

Fig. 15. Photo of the paper-based prototype.

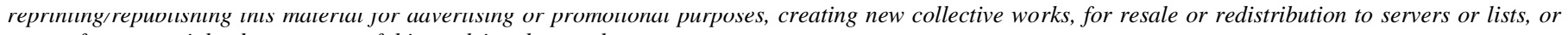
reuse of any copyrighted component of this work in other works.

doi: 10.1109/TAP.2015.2452969 
other ceramic substrates, allows it to turn into organic matter in landfills in only a few months [32]. For this purpose a scaled version of the proposed antenna has been designed and realized on a 0.69 mm-thick paper substrate $\left(\varepsilon_{\mathrm{r}}=2.85\right.$, $\tan (\delta)=0.053 @ 4 \mathrm{GHz})$. This first prototype is realized by hand-painting a conductive paste over a thin cardboard. The photo of the paper-based prototype is shown in Fig. 15 together with its final layout dimension: a scaling factor of 1.22 with respect to the prototype of Fig. 8 is adopted, taking into account the different substrates permittivity. For measurement purposes we make use of a conductive glue in the connection between the coaxial junction and the paper prototype.

The behavior of the two prototypes is very similar. In particular the normalized radiation patterns of Figs. 12-13 pertain to the paper antenna, too, and are not reported here for the sake of brevity. Fig. 16 reports the comparison between measured and modelled input impedance of the paper-based antenna: the results are in almost perfect agreement in the UHF band (in particular the $1.5-\lambda$ resonant behavior at 868 $\mathrm{MHz}$ ), while some differences are observed in the UWB band, probably due to the imperfect metallization.

In Tab. I the predicted radiation efficiencies all over the frequency bands are compared for a lossy and lossless paper. One drawback of the paper solution is the modest efficiency in the UHF band: the long and winding current path at $868 \mathrm{MHz}$ is responsible for an efficiency of $63 \%$ (almost $70 \%$ is the corresponding value on a FR-4 support). Higher values are observed in the UWB band due to the shorter current path of the circular "active zone" when frequency values greater than

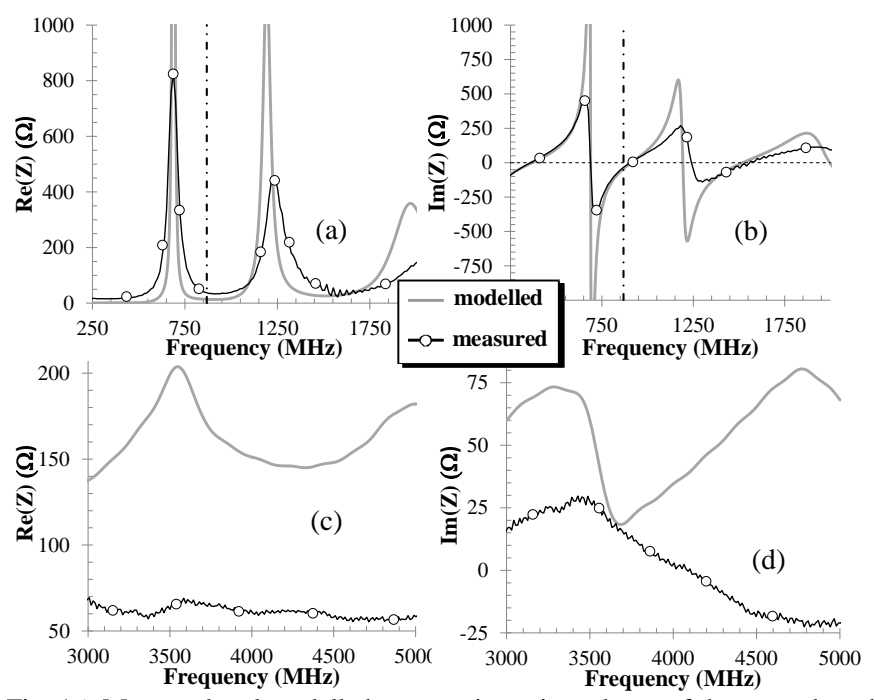

Fig. 16 Measured and modelled antenna input impedance of the paper-based prototype of Fig. 15: (a) real part, (b) imaginary part in the UHF band; (c) real part, (d) imaginary part in the UWB band.
$3 \mathrm{GHz}$ are involved (see Fig. 4). The higher the frequency, the shorter the active part of the loop, and the higher the efficiency.

A final test of the link performance has been carried out in a real indoor scenario using the two prototypes. We measured the power received by a horn antenna (TDK HRN-0118) with broadband and medium-gain capabilities, located at $3 \mathrm{~m}$ distance from our antennas in broadside $\left(\theta=0^{\circ}\right)$ transmitting mode. Similar values with respect to simulated results have been obtained: at the UWB center frequency $(4 \mathrm{GHz})$ the measured gain of the FR-4 antenna is $2.9 \mathrm{dBi}$, while in the same conditions the paper-based antenna shows a 2-dB reduction.

\section{THREE-PORT ANTENNA FEEDING NETWORK DESIGN}

In this section we describe the design of a three-portmicrostrip/lumped element antenna feeding network able to simultaneously provide high decoupling between the UHF and the UWB paths and the matching conditions of the two paths. The challenging aspect is to reach these goals with reduced dimensions in order not to affect the antenna performance. In view of a unique UWB-UHF chip realization, this network could be used as a proof-of-concept to be integrated in a unique chip.

Different aspects need be simultaneously accounted for in this feeding network design, namely: i) preserving the radiating performance of the ungrounded antenna; ii) realizing a suitable transition from the antenna balanced port to the unbalanced microstrip network; iii) separating and matching the UHF and UWB paths with minimum insertion losses.

In Fig. 17(a) the back view of the proposed non-invasive feeding solution is shown (here the antenna paper substrate is made transparent for visualization purposes): it consists of a 1$\mathrm{cm}^{2}$ brick of paper, $2.5 \mathrm{~mm}$-thick, attached to the back side of the antenna. A balanced line departing from the antenna is realized inside the brick. In this way we realize the transition from a horizontal (the antenna) to a vertical (new couple of strips) balanced line: in Fig. 17(b) a zoomed view of this transition is shown, with the $1-\mathrm{cm}^{2}$ paper brick removed for ease of visualization. The balanced line output is connected to a vertically-aligned planar microstrip circuit (its ground plane is visible in the figure), with a paper sheet as substrate, having the same thickness of the antenna sheet $(0.69 \mathrm{~mm})$. The total area of the three-port network is only of $12 \times 10 \mathrm{~mm}^{2}$. This miniaturized solution allows an effective balun-free transition from balanced-to-unbalanced architectures [33, 34] and guarantees quite unaltered antenna performance both in terms of radiation patterns and polarization, all over the frequency bands of interest. 
Nonlinear/electromagnetic co-simulation is then adopted to optimize the network for the stringent design goals described above. As a realistic load for the UHF output we have adopted a standard full-wave nonlinear rectifier (for energy harvesting purposes); while the UWB-port is conventionally loaded by a $50 \Omega$ termination.

We have proved that the procedure can be successfully followed for different combinations of loading conditions, depending on the specific components choice for the UHF and the UWB branches. For the present case, the resulting network topology is schematically described in Fig. 18(a): the UWB path consists of a double-stub filter, while the UHF path is realized by a T-network of lumped SMD components whose values are: $\mathrm{L}_{\mathrm{s} 1}=0.1 \mathrm{nH} ; \mathrm{C}_{\mathrm{s} 1}=19 \mathrm{pF} ; \mathrm{L}_{\mathrm{p} 2}=1.1 \mathrm{nH} ; \mathrm{C}_{\mathrm{p} 2}=38 \mathrm{pF}$; $\mathrm{L}_{\mathrm{s} 3}=82 \mathrm{nH} ; \mathrm{C}_{\mathrm{s} 3}=1 \mathrm{pF}$. The corresponding meandered adopted layout on paper substrate is shown in Fig. 18(b). The rectifier is a standard voltage doubler with two Skyworks SMS7630 diodes, loaded by the optimum load (20 $\mathrm{k} \Omega$, in this case), [8].

The simulated 3-port circuit behavior is reported in Fig. 19, in terms of scattering-parameter amplitudes: in this case a complex impedance of $20-\mathrm{j} 250$ is used as the UHF port impedance. This constant value has to be understood as an average of the variable non-linear rectifier input impedance at the expected low input power levels (from $-20 \mathrm{dBm}$ to $0 \mathrm{dBm}$ ). For the present purpose the UWB port has been kept referenced to $50-\Omega$. The $S_{21}$ and $S_{31}$ plots show promising values of the transmission coefficients between the antenna port and the UHF and UWB ports, respectively: they are equal or greater than $-2 \mathrm{~dB}$ in both cases and all over the bands of interests, in spite of the high paper losses. The effectiveness of the designed network is also confirmed by the high decoupling between the UHF and the UWB ports all over the bands (see $\mathrm{S}_{23}$ in Figs. 19(a) and (b)). The realized paper-based prototype is measured in its final configuration, i.e. with port 2 loaded

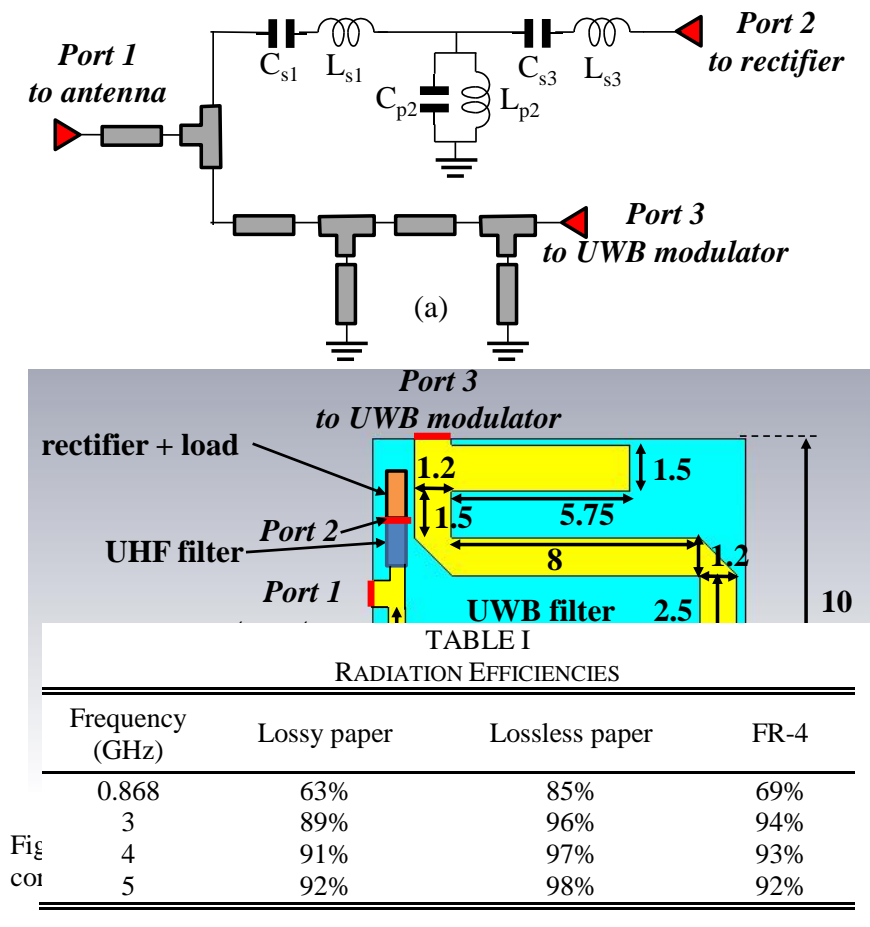

reuse of any copyrighted component of this work in other works. doi: 10.1109/TAP.2015.2452969

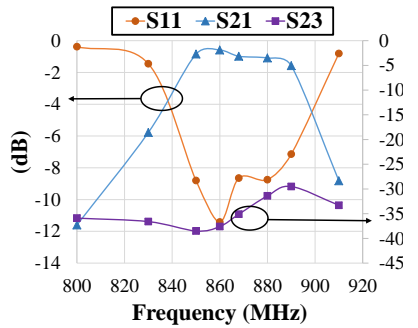

(a)

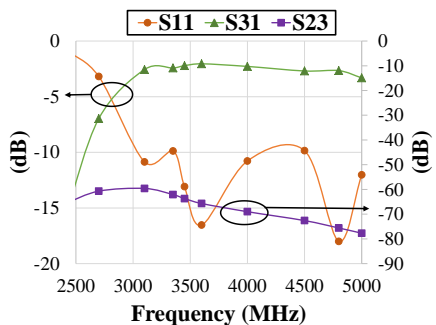

(b)
Fig. 19. Simulated scattering parameters of the three-port feeding network of Fig. 18(a): (a) in the UHF band; (b) in the UWB band.

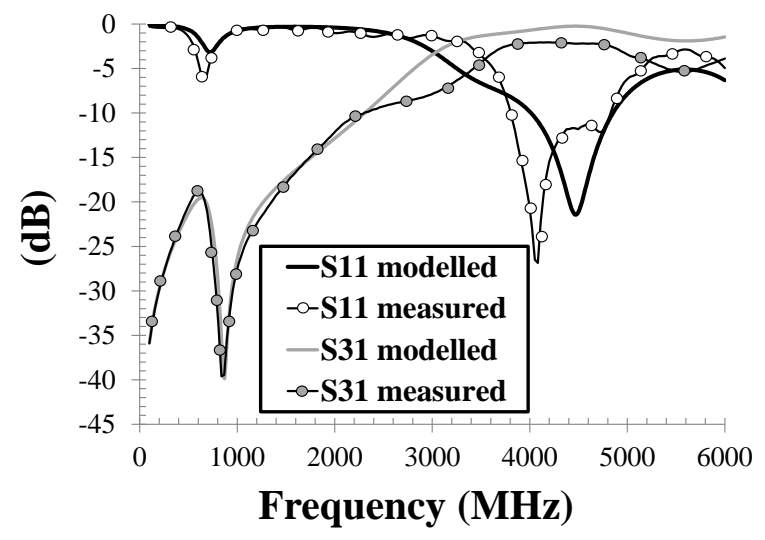

Fig. 20. Measured and modelled scattering parameters of the feeding network loaded by the rectifier (as in Fig. 18(b)) (normalized to $50 \Omega$ ).

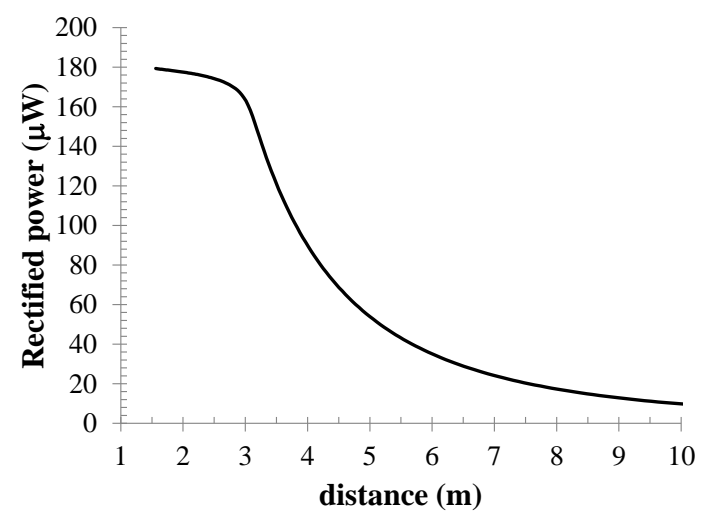

Fig. 21. Rectified power from a 868-MHz dipole source with $2 \mathrm{~W}$ ERP, as a function of distance.

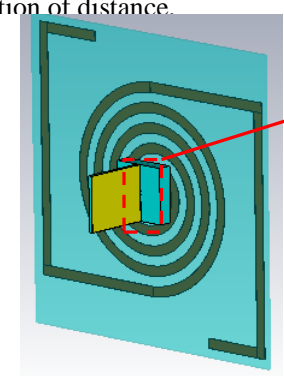

(a)

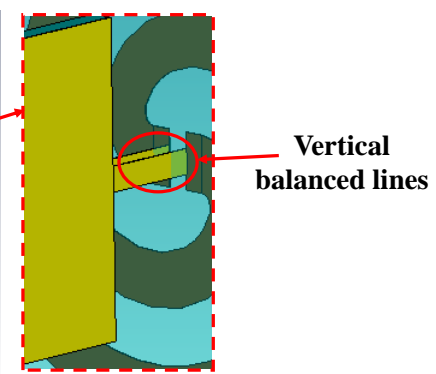

(b)
Fig. 17. (a) Perspective back-side view of the new antenna with proposed feeding network; (b) detail of the feeding network-antenna connection (brick of paper removed for ease of visualization).

by the rectifier, and thus no more directly accessible. The 
corresponding measurement shows good agreement with the model, as demonstrated by the comparison in terms of scattering parameters between port 1 and port 3, reported in Fig. 20: it is noteworthy that, for measurement setup needs, these parameters are normalized to $50 \Omega$ to comply with the network analyzer input impedance.

Finally an estimation of the rectified power on the optimum load resistance of the UHF-rectifying circuit is reported in Fig. 21. A 2-W ERP for a standard dipole resonating at $868 \mathrm{MHz}$ is considered at the RF source side: about $50 \mu \mathrm{W}$ of DC power is predicted at a distance of $5 \mathrm{~m}$, which is compatible with the power consumption of ultra-low power sensors. The same setup is used to give an estimation of the system reading range capabilities, considering the typical power consumptions of available tags chips [35] : if an average of $-15 \mathrm{dBm}$ is adopted as a threshold activation power level, a distance of about $4.5 \mathrm{~m}$ can be covered by the system under consideration.

\section{CONCLUSION}

The proposed antenna system introduces for the first time a novel, single-port, compact, low-profile antenna capable of hybrid UWB-UHF operations. This miniaturized, ecocompatible architecture has dimensions suitable for current and future RFID applications. It allows to exploit UWB technology, for accurate localization and communication, and UHF band for efficient energy harvesting, while preserving compatibility with previous RFID generations. Design steps have been described and justified, and numerically validated by EM simulation. Measurements in real environments have shown similar performance over both the 3.1 to $4.8 \mathrm{GHz}$ UWB band and the $868 \mathrm{MHz}$ UHF-RFID band.

This efficient, compact, low-profile antenna is thus foreseen as a potential candidate for future eco-friendly zero-power smart tags: in spite of the not negligible losses of the paper substrate, the antenna performance are fully compatible with UWB communication and UHF energy harvesting requirements. Exploitation of alternative recyclable materials, introducing lower losses (e.g., PET, fabrics), can be straightforwardly addressed by simply scaling the antenna layout.

The proposed UWB-UHF antenna system is thus ready to be adopted by a customized subsystem realizing UHF rectification and UWB backscattering communication [10].

\section{ACKNOWLEDGMENT}

This work was partly funded by the Italian Ministry of the Instruction, University and Research (MIUR), within the framework of the national project GRETA ("GREen TAgs and sensors with ultra-wideband identification and localization capabilities") and by EU COST Action IC1301 "Wireless Power Transmission for Sustainable Electronics (WIPE)"..

The authors would like to thank Eng. Nicola Arbizzani for his valuable contribution in measurement procedures.

\section{REFERENCES}

[1] D. Dardari, R. D’Errico, C. Roblin, A. Sibille, and M. Win, "Ultrawide Bandwidth RFID: The next Generation?," Proc. of the IEEE, vol. 98, no. 9, pp. 1570-1582, Aug. 2010.

[2] V. Heires, K. Belmkaddem, F. Dehmas, B. Denis, L. Ouvry, R. D'Errico, "UWB Backscattering System for Passive RFID Tag Ranging and Tracking", Int. Conf. on Ultra-Wideband, 2011, pp. 489-493.

[3] Zhou Yuan, C.L. Law, Xia Jingjing, "Ultra low-power RFID tag with precision localization using IR-UWB," 2011 IEEE MTT-S Int. Microwave Symp. Digest, , pp.1-4, June 2011.

[4] G. Selimis, J. Romme, H. Pflug, K. Philips, G. Dolmans, H. de Groot, "Sub-meter UWB localization: Low complexity design and evaluation in a real localization system," 2013 IEEE 24th Int. Symp. on Personal Indoor and Mobile Radio Communications, pp.186-191, Sept. 2013.

[5] M. Baghaei-Nejad, D.S. Mendoza, Zou Zhuo, S. Radiom, G. Gielen, Zheng Li-Rong, H. Tenhunen, "A remote-powered RFID tag with $10 \mathrm{Mb} / \mathrm{s}$ UWB uplink and $-18.5 \mathrm{dBm}$ sensitivity UHF downlink in $0.18 \mu \mathrm{m}$ CMOS," IEEE International Solid-State Circuits Conference, pp. 198-199, Feb. 2009.

[6] F. Guidi, A. Sibille, C. Roblin, "Interaction of UWB Tag Backscattering With Close Metallic Reflectors," IEEE Antennas and Wireless Propagation Letters, vol.13, pp. 245-248, 2014.

[7] P. Kalansuriya, N.C. Karmakar, E. Viterbo, "On the Detection of Frequency-Spectra-Based Chipless RFID Using UWB Impulsed Interrogation," IEEE Trans. on Microwave Theory and Techniques, vol.60, no.12, pp. 4187-4197, Dec. 2012.

[8] D. Masotti, A. Costanzo, P. Francia, M. Filippi, A. Romani, "A LoadModulated Rectifier for RF Micropower Harvesting With Start-Up Strategies," IEEE Trans. on Microwave Theory and Techniques, vol. PP, no.99, pp.1-11, 2014.

[9] http://www.eia.org.uk/HORIZON2020.pdf.

[10] National Italian project PRIN-GRETA ("GREen TAgs and sensors with ultra-wideband identification and localization capabilities"), 2012-2015.

[11] R D'Errico, M. Bottazzi, F. Natali, E. Savioli, S. Bartoletti, A. Conti, D. Dardari, N. Decarli, F. Guidi, F. Dehmas, L Ouvry, U. Alvarado, N. Hadaschik, N. C. Franke, Z. Mhanna, M. Sacko, Y. Wei, A. Sibille, "An UWB-UHF semi-passive RFID System for localization and tracking applications," IEEE Int. Conf. on RFID-Technologies and Applications, 2012, Nov. 2012, pp.18-23.

[12] C.C. Cruz, J.R. Costa, C.A Fernandes, "Hybrid UHF/UWB Antenna for Passive Indoor Identification and Localization Systems," IEEE Trans. Ant. and Prop., Vol. 61, no. 1, 2013, pp. 354-361.

[13] A. Ramos, A. Lazaro, D. Girbau, "Semi-Passive Time-Domain UWB RFID System," IEEE Trans. Microwave Theory and Techniques, Vol.61, no.4, April 2013, pp.1700-1708.

[14] M. Pigeon, R. D'Errico, C. Delaveaud, "UHF-UWB tag antenna for passive RFID applications," 7th European Conference on Antennas and Propagation (EuCAP), 2013, pp. $3968-3972$.

[15] V. Rizzoli, A. Costanzo, and P. Spadoni, "Computer-Aided Design of Ultra-Wideband Active Antennas by Means of a New Figure of Merit," IEEE Microwave Wireless Comp. Lett., Vol. 18, No. 4, Apr. 2008, pp. 290-292.

[16] Y. Lu, Y. Huang, Y.C. Shen, H.T. Chattha, "A further study of planar UWB monopole antennas," Antennas \& Propagation Conference (LAPC), 2009, pp. $353-356$.

[17] W. Wiesbeck, G. Adamiuk, C. Sturm, "Basic Properties and Design Principles of UWB Antennas," Proc. of the IEEE, Vol. 97 , no. 2, 2009, pp. $372-385$.

[18] E. Gazit, "Improved design of the Vivaldi antenna", Proc. of the IEEE. Vo1. 135, Pt.H, no.2, April 1988, pp. 89-92.

[19] W.E. Stutzman, G.A. Thiele, "Broadband Antennas," in Antenna Theory and Design, 2nd ed. Wiley \& Sons. INC, 1998.

[20] O. Ahmad Mashaal, S.K.A. Rahim, A.Y. Abdulrahman, M.I. Sabran, M.S.A. Rani, P.S. Hall, "A Coplanar Waveguide Fed Two Arm Archimedean Spiral Slot Antenna With Improved Bandwidth," IEEE Trans. Antennas and Propagation, vol.61, no.2, pp. 939-943, Feb. 2013.

[21] A.R. Guraliuc, R. Caso, P. Nepa, J.L. Volakis, "Numerical Analysis of a Wideband Thick Archimedean Spiral Antenna," IEEE Antennas and Wireless Propagation Letters, vol.11, pp.168-171, 2012.

[22] M. Lee, B.A. Kramer, C.C. Chen, J.L. Volakis, "Broadband spiral antenna miniaturization limit", Antennas and Propagation Society Int. Symposium 2006, pp. 3701-3704.

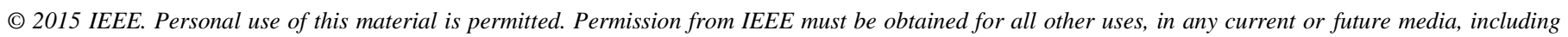

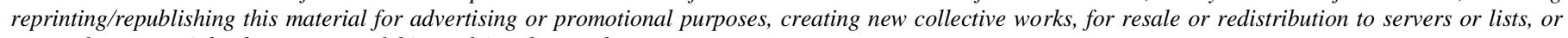
reuse of any copyrighted component of this work in other works.

doi: 10.1109/TAP.2015.2452969 
[23] B.A. Kramer, C.C. Chen, M. Lee, J.L. Volakis, "Fundamental Limits and Design Guidelines for Miniaturizing Ultra-Wideband Antennas", Ant. and Prop. Magazine, Vol. 51, no. 4, 2009, pp. 57-69.

[24] B.A. Kramer, M. Lee, C.C. Chen, J.L. Volakis, "Design and performance of an ultrawide-band ceramic-loaded slot spiral", IEEE Trans. Ant. and Prop., Vol. 53, no.7, 2005, pp.2193-2199.

[25] M. Aldrigo, A. Costanzo, D. Masotti, C. Baldisserri, I. Dimitru, C. Galassi, "Numerical and experimental characterization of a buttonshaped miniaturized UHF antenna on magneto-dielectric substrate", Int. J. Microwave And Wireless Technologies, Vol 5, No.3., pp. 231-239, June 2013.

[26] Chaoyuan Ding, Chengli Ruan, Ling Peng, Jiahui Chu, "A novel archimedean spiral antenna with uniplanar EBG substrate," 8th Int. Symp. on Ant. Prop. and EM Theory, (ISAPE), 2008, pp. 313 - 315

[27] CST Microvawe Studio 2013, www.cst.com.

[28] K.D. Palmer, M.W. van Rooyen, "Simple broadband measurements of balanced loads using a network analyzer," IEEE Trans. Instrum. and Meas., Vol. 55 , no. 1, 2006 , pp. $266-272$.

[29] J. Essel, D. Brenk, J. Heidrich, R. Weigel, D. Kissinger, "Large-Signal Measurements and Nonlinear Characterization of an Analog Frontend for Passive UHF CMOS RFID Transponders," IEEE Trans. Microwave Theory and Techniques, Vol.61, no.2, pp. 948-959, Feb. 2013.

[30] K. Hirose, M. Miyamoto, H. Nakano, "A two-wire spiral antenna with unbalanced feed," IEEE Antennas and Propagation Society International Symposium, Vol.4, July 2001, pp.128-131.

[31] R.K. Tallos, Wang Zheyu, J.L. Volakis, "Wi-Fi energy harvesting system using body-worn antennas," IEEE Antennas and Propagation Society International Symposium (APSURSI), July 2014, pp.1405-1406.

[32] V. Lakafolis, A. Rida, R. Vyas, L. Yang, S. Nikolaou and M.M. Tentzeris, "Progress Towards the First Wireless Sensor Networks Consisting of Inkjet-Printed, Paper-Based RFID-Enabled Sensor Tags", Proc. IEEE, Sept. 2010.

[33] M. Del Prete, D. Masotti, N. Arbizzani, and A. Costanzo, "Remotely Identify and Detect by a Compact Reader With Mono-Pulse Scanning Capabilities", IEEE Trans. Microwave Theory and Techniques, Vol. 61, No. 1, Part II, Jan. 2013, pp. 641-650.

[34] E.A. Navarro, J.M. Blanes, J.A. Carrasco, C. Reig, "Yagi-Like Printed Antennas for Wireless Sensor Networks", IEEE Int. Conf. Sensor Tech. and Applications, 2007, pp. 254-259.

[35] K.V.S. Rao, P.V. Nikitin, S.F. Lam, "Antenna design for UHF RFID tags: a review and a practical application," IEEE Trans. Antennas and Propagation, Vol.53, no.12, Dec. 2005, pp. 3870-3876.

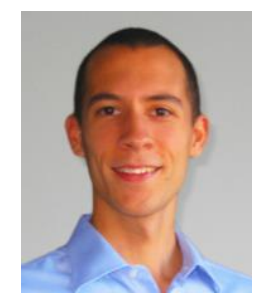

Marco Fantuzzi received the B.S. degree from the University of Modena and Reggio Emilia, Modena, Italy, and the M.S. degree from the University of Bologna, Bologna, Italy, in 2010 and 2013, respectively.

He joined the Department of Electrical, Electronic and Information Engineering of the University of Bologna in 2014, where he is currently working toward the Ph.D. degree.

His research interests include the design of RF energy harvesting and Wireless Power Transfer systems, as well as RFID technologies and UWB systems.

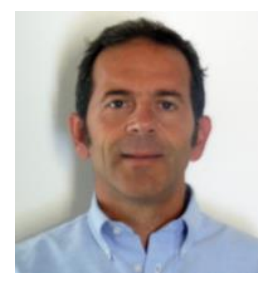

Diego Masotti ( $\left.\mathrm{M}^{\prime} 00\right)$ received the Dr. Ing. degree in electronic engineering and Ph.D. degree in electric engineering from the University of Bologna, Bologna, Italy, in 1990 and 1997, respectively.

In 1998 he joined the University of Bologna as a Research Associate of electromagnetic fields. His research interests are in the areas of nonlinear microwave circuit simulation and design (with emphasis on modern CAD techniques for large-size problems). More recently Dr. Masotti has devoted his research activity to circuital/electromagnetic co-design of nonlinear radiating subsystems/systems, with special attention to energy harvesting and near/far-field wireless power transfer applications. He has co-authored more than 120 scientific publications on peer reviewed International Journals and conferences, and two book chapters.

Dr. Masotti has been a member of the Paper Review Board of the IEEE TRANSACTIONS ON MICROWAVE THEORY AND TECHNIQUES, IEEE Communication Letters, IET-Circuit Devices \& Systems, IEEE MICROWAVE AND WIRELESS COMPONENTS LETTERS, since 2004, 2010, 2011, and 2013, respectively.

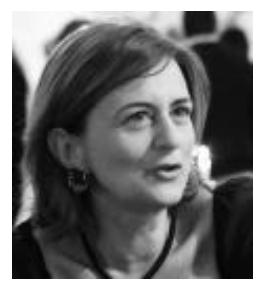

Alessandra Costanzo received a Doctor degree in Electrical Engineering (magna cum laude) from the University of Bologna, Italy. Since 2001, she has been Associate Professor of electromagnetic fields at the University of Bologna, Polo di Cesena. She has co-authored more than 130 scientific publications on peer reviewed International Journals and conferences and three book chapters. She holds three international patents. Her research activities have focused on several topics such as: electro-thermal characterization and modeling of $\mathrm{RF} /$ microwave nonlinear devices; simulation and design of active microwave integrated circuits; broadband design of self-oscillating circuits and systems for electrical, stability, and noise performance. More recently, Prof. Costanzo has worked on an innovative software platform for the nonlinear and electromagnetic co-simulation of RF systems, excited by modulated sources. She has demonstrated the circuit-level analysis of entire MIMO and UWB links, including realistic channel models. She is currently involved in the development of energy autonomous sensors based on wearable systems and energy harvesting technologies and of wireless power transfer systems. Many are her research and industrial collaborations that have led to significant funding sources from both institutional and private investors.

She is member of the management committee of the recently approved EU COST action WiPE "Wireless power transfer for sustainable electronics". She is member of the TPC board of the MTT-S IMS and of the EUMW and of the steering committee of the 2014 EUMW, which will be held in Italy. She is chair of IEEE MTT-S TC-26 Wireless Energy Transfer and Conversion and member of IEEE MTT-S TC-24 RFID Technologies. She serves at the Editorial board of many international journals such as IEEE-T-MTT, IEEE-TCAD, IEEE MWGL and IJMWT. She is executive editor of the Cambridge University Press Wireless Power Transfer Journal. 